\title{
A RIGHT-SIDED TRUE DIAPHRAGMATIC HERNIA WITH UNUSUAL FEATURES
}

BY

GLADYS H. DODDS, M.D., D.P.H.,

and

\author{
J. A. POCOCK, L.R.C.P., M.R.C.S.
}

(From the Obstetric Unit, University College Hospital, London.)

Congenital diaphragmatic hernia is a rare and grave condition. It is well known as a cause of still-birth and neonatal death. Of 127 cases reviewed by J. S. Latta ${ }^{1}$ the age was given in 112 cases and of these 88 were either stillborn or died within a few hours of birth. In a series of 57 cases collected by Funck Brentano 51 cases were either still-born or died within 24 hours of birth.

The term diaphragmatic hernia is applied to any protrusion of the abdominal contents through the diaphragm. In a majority of the cases the abdominal contents pass through an opening in the diaphragm not constituting a hernia in the true sense of the word but a spurious diaphragmatic hernia. The true diaphragmatic hernia projects into the pleural cavity through a space in the muscle covered by peritoneum and pleura. Among 80 cases of congenital hernia collected by Bohn ${ }^{3}$ a hernial sac was present in only 14. The left side is more frequently affected than the right. Of 42 cases of diaphragmatic hernia according to Popp 4 , 37 were on the left side and only 5 on the right. Of Latta's 127 cases only 17 were on the right side. Further, when the right side is affected the hernia is usually small. Spencer ${ }^{5}$ records two cases of large right-sided diaphragmatic herniæ. According to Ballantyne ${ }^{6}$ the male sex seems to be more liable to diaphragmatic hernia than the female ; thus in 67 case records examined by him 47 were males and 20 females. Only occasionally is the diaphragmatic defect accompanied by other developmental defects, except that of an undeveloped lung, and in practically all cases the child is normal in external appearance.

The following case is recorded not only on account of the rarity of the large right-sided true diaphragmatic hernia, but also because of the associated mal-development of suprarenal gland and secondary herniation of the primary sac.

\section{Case report.}

Baby B., female, was born on the district of the Obstetric Hospital, University College Hospital, a few minutes before the arrival of the attendants. The baby was blue, cried feebly and gasped about four times to the minute. It lived for nearly half an hour. The mother was a healthy woman aged 33 years. Her Wassermann reaction was negative. She had had 6 previous children, all of whom were alive and healthy and showed no deformity. 
Post-morten exanination.-Full-time, female child, weight 3,200 grm., length $49 \mathrm{~cm}$. Externally the child showed no deformity. On opening the thoracic cavity a large solid structure similar in appearance to the liver was noted lying within a sac in the right pleural cavity. On opening the sac this lobe of liver was found to occupy the entire anterior part of the right side of the thorax. It corresponded in shape to the thorax, the inner left margin was almost vertical and reached an apex at the level of the 3rd rib, where the right margin sloped outwards towards the ribs. On the postero-lateral surface of this lobe of the liver the gallbladder was recognized, attached closely to the liver. The apex of the gall-bladder reached the antero-lateral margin of the liver at the level of the 4 th rib in the mid-axillary line. The gall-bladder lay in an almost vertical plane, hence the neck of the gall-bladder was close to the opening in the diaphragm. Posterior to the liver lay intestines. The right lung was undeveloped but showed normal lobulation, and lay against the thoracic vertebræ posterior to the hernial sac. The heart was pushed over into the left thorax, but was otherwise normal. The left lung was small and undilated, but normal in shape and fissuring. The thymus was small, weighing 2 grm.

On opening the abdominal cavity the liver and large intestine presented. The abdominal liver had a right and a left lobe. The left loce was the larger of the two and was normal in shape. The right lobe was almost square and was about half the size of the left lobe. On the inferior surface of the right lobe a caudate lobe of normal size was seen in its normal position, but quadrate lobe, portal tract and gall-bladder were absent. The gastro-hepatic omentum extended from the depression on the liver anterior to caudate lobe on to the lesser curvature of the stomach. The stomach was normal in size and position. On raising the abdominal portion of the liver an aperture which admitted 3 fingers was seen in the antero-lateral aspect of the right side of the diaphragm. A sac consisting of peritoneum and pleura extended into the thorax from the margins of the hernial opening. A narrow neck of liver joining the abdominal and thoracic portions lay in the anterior part of the hernial opening; posteriorly were intestines.

The pylorus lay on the right side of the first lumbar vertebra and from it the first part of the duodenum passed upwards posteriorly and slightly to the left into the thorax, where it lay in close proximity to the neck of the gall-bladder. The second part of the duodenum curved laterally from the neck of the gall-bladder. The third part of the duodenum passed almost directly caudally into the abdominal cavity where it lay in direct contact with the posterior abdominal wall in the plane of right mid-axillary line. The head of the pancreas lay in the curvature of the duodenum. The second part of the duodenum was free, i.e., it was covered by peritoneum on all surfaces except where the head of the pancreas lay in contact with it. The common bile duct and pancreatic duct entered the second part of the duodenum.

The jejunum arose from the duodenum in the abdomen and passed immediately into the thoracic cavity posterior to the lobe of the liver. The small intestine filled the posterior area of the hernial sac. There was a secondary hernial sac, situated on the lower postero-medial aspect of the primary hernial sac, and this projected medially into the posterior mediastinum. The lower end of ileum, cæcum and appendix were found in this secondary hernial sac. The mesentery of the small intestine was attached to the posterior abdominal wall inferior to the posterior margin of the hernial opening; thus it was slightly superior and more lateral than normal. The ascending colon passed laterally from the posterior mediastinum into the abdominal cavity and became the transverse colon close to the pylorus. The ascending colon had a mesentery which was attached to the greater curvature of the stomach. The transverse, descending and pelvic colons were normal. Both kidneys, spleen and left suprarenal were normally situated. The right suprarenal was very small in size, and in weight was less than $1 \mathrm{grm}$. Microscopical examination of it revealed normal structure. The head of the pancreas was in the duodenal curvature in the thoracic cavity but the body and tail were normally situated in the abdomen, in relation to the stomach and spleen. There was no cerebral nor osseous deformity.

\section{Discussion.}

The development of the diaphragm is a complicated process. It is built up of 5 separate parts-one central, two ventro-lateral and two dorsi-lateral. At about the 6th week of embryonic life after the heart, lungs, liver and stomach 
are in their permanent positions, the openings between the pleural and peritoneal cavities close and about the same time muscle fibres are laid down in the membranes which are forming the diaphragm. The true congenital diaphragmatic hernias are due to an abnormal development of the diaphragmatic muscle and any process which retards or prevents this may easily lead to a herniation at the point of defect. Why the muscle fails at times to develop is not certain. It may be a question of local pathology-some interference in circulation or some fault in innervation. In this case the phrenic nerves were present on each side, the left innervating the left side of the diaphragm and the right being distributed to the hernial sac on the right side.

\section{Summary.}

This case shows a combination of rare features :-(1) a large right-sided true diaphragmatic hernia in a female ; (2) abnormal fissuring of the liver and situation of the gall-bladder; (3) the duodenum entirely confined to the right side of the body and not closely attached to the posterior abdominal wall; (4) a mesentery attached to the ascending colon ; (5) a secondary herniation of the primary sac ; (6) a rudimentary suprarenal gland.

We are indebted to Professor F. J. Browne, Director of the Obstetric Unit, University College Hospital, for permission to publish this case.

\section{REFERENCES.}

1. Latta, J. S., Am. J. Dis. Child., Chicago, 1922, XXIV, 297.

2. Funck-Brentano, quoted by Rhenter, J. La pratique de l'art accouchements, 1928, V, 249.

3. Bohn, quoted by Tillmanns, H. Lehrb. der spez. Chirug., 1904, I, 640.

4. Popp, quoted by Birnbaum \& Blacker. Malformations of the foetus, 1912, 145.

5. Spencer, H., Trans. Obst. Soc., Lond., 1890, XXXII, 132.

6. Ballantyne, J. W., Antenatal Path. \& Hyg., The Embryo, Edin., 1904, 483. 